\title{
Novel insights into the role of aberrantly expressed MNX1 (HLXB9) in infant acute myeloid leukemia
}

\author{
Juerg Schwaller \\ University Children's Hospital beider Basel (UKBB), Department of Biomedicine, University of Basel Childhood Leukemia Group ZLF, \\ Switzerland.
}

E-mail:J.Schwaller@unibas.ch

doi:10.3324/haematol.2018.205971

A Imost two decades ago, the molecular characterization of a $\mathrm{t}(7 ; 12)(\mathrm{q} 36 ; \mathrm{p} 13)$ chromosomal translocation in very young children with acute myeloid leukemia (AML) and poor outcome identified a fusion mRNA potentially encoding for a chimeric protein that contains the pointed (PNT) and ETS domains of the ETS variant 6 (ETV6) gene, also known as TEL1 (Translocating E26 transforming-specific leukemia 1) on 12p13, joined to the regulatory sequences and first exons of the HLXB9 homeobox gene. ${ }^{1}$ Previous work reported a series of infant AML patients with $t(7 ; 12)(q 36 ; p 13)$ with blasts carrying a potential ETV6 translocation (revealed by a split FISH signal). ${ }^{2,3}$ In fact, the entire HLBX9 gene seems to be transferred onto the der(12) without disruption of the gene itself. A fusion transcript may result from a yet to be identified long-range splicing mechanism and can be found in about $50 \%$ of cases. Expression of any reciprocal ETV6HLXB9 fusion transcript has not been reported. These findings suggested that a position effect triggers stable overexpression of HLXB9 in $\mathrm{t}(7 ; 12)(\mathrm{q} 36 ; \mathrm{p} 13)^{+}$AML. ${ }^{4,5}$ The Nordic Society for Pediatric Hematology and Oncology (NOPHO) recently reported additional 7 patients to the previously 35 published $\mathrm{t}(7 ; 12)(\mathrm{q} 36 ; \mathrm{p} 13)^{+} \mathrm{AML}$ patients. Overall leukemic blasts from $20-30 \%$ of infant AML patients ( $<2$ years) carry this translocation associated with trisomy 19 in $86 \%$ of the cases. ${ }^{6}$ In addition to $\mathrm{t}(7 ; 12)(\mathrm{q} 36 ; \mathrm{p} 13)$, another translocation, $\mathrm{t}(6 ; 7)(\mathrm{q} 23 ; \mathrm{q} 35)$, was identified in an AML cell line (GDM-1) which also resulted in aberrantly high HLXB9 expression via juxtaposition with regions of the MYB gene.?

The homeobox HLXB9 (or HB9) protein is today referenced as MNX1 (motor neuron and pancreas homeobox 1, OMIM: 142994). Putative loss-of-function mutations of MNX1 were identified as the molecular correlates of hereditary sacral agenesis. ${ }^{8}$ Gene targeting experiments in mice revealed MNX1 to be a critical regulator for normal pancreas ${ }^{9,10}$ as well as motor neuron development. ${ }^{11}$ Loss-of-function $M N X 1$ mutations in neonatal diabetes confirmed its role in pancreas development in humans. ${ }^{12}$

There is increasing evidence to suggest an important role of this critical developmental homeobox transcription factor not only in hematologic malignancies but also in solid cancers; ${ }^{13,14}$ however, the functional consequences and molecular mechanisms of aberrantly high MNX1 expression for malignant transformation remain poorly understood.

In a study published in this edition of Haematologica, Ingenhag et $a l^{15}$ addressed the oncogenic potential of increased MNX1 expression in non-hematopoietic and hematopoietic cells. They found that lentiviral MNX1 overexpression in human HT1080 fibrosarcoma or mouse NIH3T3 fibroblasts resulted in growth arrest with stalling at the G1/G2 phase of cell cycle, morphological signs of senescence, and increased senescence-associated $\beta$-galactosidase activity (SA- $\beta$-gal) associated with activation of the tumor suppressor
TP53 and its target the cyclin-dependent kinase inhibitor 1A (CDKN1A, aka p21 $\left.{ }^{\mathrm{WAF} 1 / \mathrm{CIP} 1}\right)$. As oncogene-induced senescence is a hallmark of early malignant transformation of solid tumors, this finding suggests that MNX1 overexpression may result in a pre-cancerous state. ${ }^{16}$ However, one has to keep in mind that both of the models used are immortalized solid cancer cell lines that may carry potent oncogenes such as mutated NRAS ${ }^{\text {Q61K }}$ present in HT-1080 (https://portals.broadinstitute.org/ccle/page?cell_line $=\quad$ HT1080_SOFT_TISSUE). Nevertheless, previous work has shown that overexpression of well-characterized AML-associated fusion oncogenes (e.g. PML-RARA, RUNX1-ETO, CBFB-MYH11) induces DNA damage, and activates a CDKN1A-dependent cell cycle arrest and DNA repair in mouse hematopoietic stem and progenitor cells (HSPC). ${ }^{17}$ It will, therefore, be interesting to verify whether overexpression of MNX1 may also lead to DNA damage activating the TP53-CDKN1A pathway in HSPC.

To address the oncogenic potential in hematopoietic cells, Ingenhag et al.$^{15}$ also lentivirally expressed MNX1 in lineage marker-depleted mouse bone marrow (BM)-derived HSPC. Reconstitution of lethally irradiated syngenic recipients resulted in a reduced overall peripheral blood cellularity with very low contribution to any mature $\left(\mathrm{CD} 19^{+} \mathrm{B}\right.$ cells; $\mathrm{CD}^{+} \mathrm{T}$ cells; CD11 $\mathrm{b}^{+}$neutrophils) cell lineage. Although the total cell number was also reduced in the BM, MNX1 expressing cells mostly contributed to the megakaryocytic-erythrocyte progenitor (MEP) cell compartment, whereas no distinct MNX1 expressing population was detected within the granulocytemonocyte progenitor (GMP) compartment. Although some MNX1 expressing immature B cells $\left(\mathrm{B} 220^{+} \mathrm{CD} 19^{+} \mathrm{CD} 93^{+}\right)$ were found, no signal for MNX1 expression was found in mature $B$ cells and immature or mature $T$ cells. None of the transplanted mice developed any signs of a hematologic disease during a relatively short observation period of 6.5 months. These observations show for the first time that MNX1 overexpression impacts hematopoietic differentiation of HSPC in vivo but may not be sufficient to induce a hematologic disease.

To study the potential oncogenic activity in human hematopoietic cells, Ingenhag et al..$^{15}$ were able to lentivirally express MNX1 in CD34+ $\mathrm{HSPC}$ at comparable levels to what was observed in primary $t(7 ; 12)(q 36 ; p 13)^{+}$AML cells. Gene expression profiling revealed that, in addition to $M N X 1,116$ significantly aberrantly regulated genes including de novo expression of HBZ (Hemoglobin Subunit Zeta), HBE1 (Hemoglobin Subunit Epsilon 1) SLC4A1 (Solute Carrier Family 4 Member 1), all tightly associated with erythroid differentiation. Notably, gene set enrichment analysis revealed upregulation of genes related to cell cycle progression, and downregulation of cytoskeleton organization and various intracellular signaling pathways. Previous work by the same group has already suggested that elevated MNX1 levels affect expression of genes 

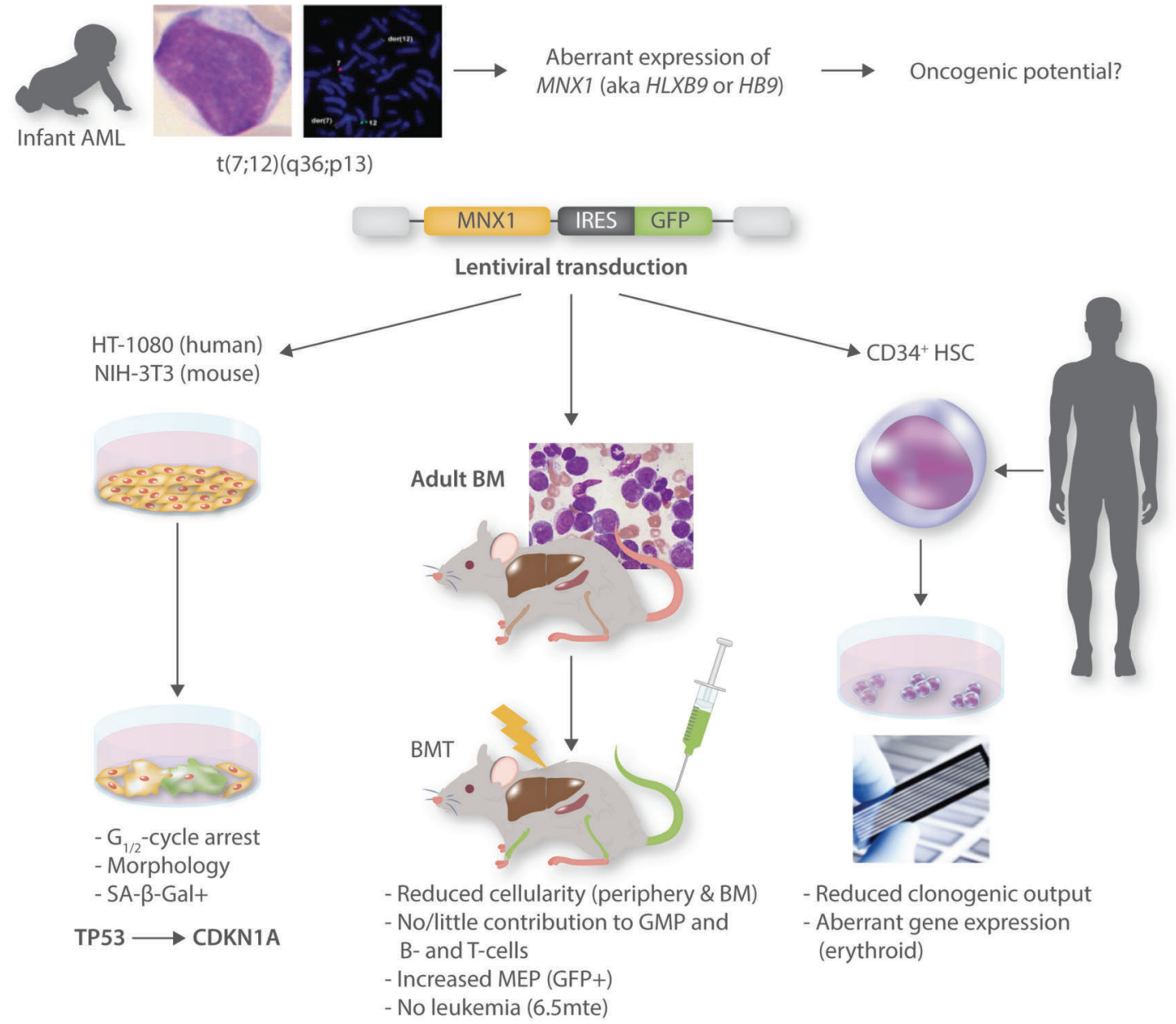

"SENESCENCE"

"IMPAIRED HEMATOPOIETIC DIFFERENTIATION"

Figure 1. Schematic representation of the findings by Ingenhag et $a l .^{15}$

implicated in cell-cell interaction and adhesion. ${ }^{18}$ Interestingly, MNX1 expression also reduced the clonogenic activity of human $\mathrm{CD} 34^{+} \mathrm{HSPC}$. Likewise previous experiments also showed no aberrant in vitro clonogenic growth of MNX1 over-expressing mouse HSPC. ${ }^{18}$ Together, the experiments in mouse and human hematopoietic cells suggest increased MNX1 expression interferes with normal hematopoietic differentiation. Notably, most of the reported infants aberrantly expressing MNX1 were diagnosed with poorly differentiated FAB subtypes. Four out of 42 published patients developed acute megakaryoblastic leukemia (AMKL) suggesting MNX1 impacts on MEP cell maturation. ${ }^{6}$

Collectively, Ingenhag et $a .^{15}$ show that aberrantly expressed MNX1 interferes with normal cycle progression, inducing premature senescence in solid cancer cell lines, and interferes with hematopoietic differentiation in vitro and in vivo (Figure 1).

This work provides important clues for our understanding of $\mathrm{t}(7 ; 12)(\mathrm{q} 36 ; \mathrm{p} 13)^{+}$infant $\mathrm{AML}$ and sets the stage for additional experiments addressing several open key questions. First, it remains to be demonstrated whether aber- rant MNX1 expression is sufficient to induce a leukemic phenotype. Although recent large deep sequencing studies did not report the presence of any common co-operating mutations (FLT3-ITD, NPM1, CEBP/A, WT1) in 4 infant AML carrying $t(7 ; 12)(q 36 ; p 13)$, the strong association with trisomy 19 in the blasts of the majority of these patients suggests some co-operative events. ${ }^{19}$ Notably, trisomy 19 has been described as a recurrent chromosomal abnormality in hematologic malignancies, including AMKL. ${ }^{20,21}$

Second, it will be important to identify the natural target cell of MNX1-mediated transformation. The exclusive association of $t(7 ; 12)(q 36 ; p 13)$ strongly suggests a fetal origin. It is somehow reminiscent of $\operatorname{inv}(16)(p 13 q 24)$ leading to expression of a CBFA2T3-GLIS2 fusion in AMKL which mostly affects infants. Modeling the transforming activity of this fusion in mice revealed that only expression in fetal liver hematopoietic cells but not in adult BM-derived cells was able to induce a leukemic disease..$^{22,23}$ Therefore, future studies will ideally follow different fetal-liver hematopoietic stem and/or progenitor cells conditionally over-expressing MNX1 in clonogenic assays and BM reconstitution assays. 
Third, what are the MNX1 target genes that mediate its leukemogenic potential? In a previous study, the same group identified binding to and regulation of the prostaglandin E receptor 2 (PTGER2) by MNX1 overexpressed in the human HL60 AML cell line..$^{24}$ However, critical targets might significantly differ in a context of fetal liver HSPC.

Finally, and most importantly, it needs to be shown whether high expression of MNX1 is critical to maintain a transformed phenotype. Knockdown or genome editing experiments in primary human AML cells (e.g. expanded in immune deficient mice) or conditional expression in transgenic mouse models may show the way. Further exploration of the MNX1 interacting proteome could provide some clues as how to develop strategies for targeted therapeutic intervention.

\section{Funding}

Our lab is supported by the Swiss Cancer League, the Swiss National Science Foundation, the Wilhelm Sander Foundation (Munich, Germany), the San Salvatore Foundation (Lugano, Switzerland), and the Novartis Research Foundation (Basel, Switzerland).

\section{References}

1. Beverloo HB, Panagopoulos I, Isaksson M, et al. Fusion of the homeobox gene HLXB9 and the ETV6 gene in infant acute myeloid leukemias with the $t(7 ; 12)(q 36 ; p 13)$. Cancer Res. 2001;61(14):5374-5377.

2. Tosi S, Harbott J, Teigler-Schlegel A, et al. t(7;12)(q36;p13), a new recurrent translocation involving ETV6 in infant leukemia. Genes Chromosomes Cancer. 2000;29(4):325-332.

3. Slater RM, von Drunen E, Kroes WG, et al. $t(7 ; 12)(q 36 ; p 13)$ and $\mathrm{t}(7 ; 12)(\mathrm{q} 32 ; \mathrm{p} 13)-$-translocations involving ETV6 in children 18 months of age or younger with myeloid disorders. Leukemia. 2001;15(6):915920.

4. von Bergh $A R$, van Drunen E, van Wering ER, et al. High incidence of $\mathrm{t}(7 ; 12)(\mathrm{q} 36 ; \mathrm{p} 13)$ in infant AML but not in infant ALL, with a dismal outcome and ectopic expression of HLXB9. Genes Chromosomes Cancer. 2006;45(8):731-739

5. Ballabio E, Cantarella CD, Federico C, et al. Ectopic expression of the HLXB9 gene is associated with an altered nuclear position in $t(7 ; 12)$ leukaemias. Leukemia. 2009;23(6):1179-1182.

6. Espersen ADL, Noren-Nystrom U, Abrahamsson J, et al. Acute myeloid leukemia (AML) with $\mathrm{t}(7 ; 12)(\mathrm{q} 36 ; \mathrm{p} 13)$ is associated with infancy and trisomy 19: Data from Nordic Society for Pediatric Hematology and Oncology (NOPHO-AML) and review of the literature. Genes Chromosomes Cancer. 2018;57(7):359-365

7. Nagel S, Kaufmann M, Scherr M, Drexler HG, MacLeod RA. Activation of HLXB9 by juxtaposition with MYB via formation of $t(6 ; 7)(q 23 ; q 36)$ in an AML-M4 cell line (GDM-1). Genes Chromosomes Cancer. 2005;42(2):170-178

8. Ross AJ, Ruiz-Perez V, Wang Y, et al. A homeobox gene, HLXB9, is the major locus for dominantly inherited sacral agenesis. Nat Genet. 1998;20(4):358-361.

9. Harrison KA, Thaler J, Pfaff SL, Gu H, Kehrl JH. Pancreas dorsal lobe agenesis and abnormal islets of Langerhans in Hlxb9-deficient mice. Nat Genet. 1999;23(1):71-75.

10. Li H, Arber S, Jessell TM, Edlund H. Selective agenesis of the dorsal pancreas in mice lacking homeobox gene Hlxb9. Nat Genet. 1999;23(1):6770 .

11. Thaler J, Harrison K, Sharma K, Lettieri K, Kehrl J, Pfaff SL. Active suppression of interneuron programs within developing motor neurons revealed by analysis of homeodomain factor HB9. Neuron. 1999;23(4):675-687.

12. Flanagan SE, De Franco E, Lango Allen $\mathrm{H}$, et al. Analysis of transcription factors key for mouse pancreatic development establishes NKX2-2 and MNX1 mutations as causes of neonatal diabetes in man. Cell Metab. 2014;19(1):146-154.

13. Desai SS, Modali SD, Parekh VI, Kebebew E, Agarwal SK. GSK-3beta protein phosphorylates and stabilizes HLXB9 protein in insulinoma cells to form a targetable mechanism of controlling insulinoma cell proliferation. J Biol Chem. 2014;289(9):5386-5398.

14. Zhang L, Wang J, Wang Y, et al. MNX1 Is Oncogenically Upregulated in African-American Prostate Cancer. Cancer Res. 2016;76(21):62906298.

15. Ingenhag $\mathrm{D}$, Reister $\mathrm{S}$, Auer $\mathrm{F}$, et al. The homeobox transcription factor HB9 induces senescence and blocks differentiation in hematopoietic stem and progenitor cells. Haematologica 2019;104(1):35-46.

16. Prieur A, Peeper DS. Cellular senescence in vivo: a barrier to tumorigenesis. Curr Opin Cell Biol. 2008;20(2):150-155.

17. Viale A, De Franco F, Orleth A, et al. Cell-cycle restriction limits DNA damage and maintains self-renewal of leukaemia stem cells. Nature. 2009;457(7225):51-56.

18. Wildenhain S, Ruckert C, Rottgers S, et al. Expression of cell-cell interacting genes distinguishes HLXB9/TEL from MLL-positive childhood acute myeloid leukemia. Leukemia. 2010;24(9):1657-1660.

19. Bolouri H, Farrar JE, Triche T Jr, et al. The molecular landscape of pediatric acute myeloid leukemia reveals recurrent structural alterations and age-specific mutational interactions. Nat Med. 2018;24(1):103-112.

20. Johansson B, Billstrom R, Mauritzson N, Mitelman F. Trisomy 19 as the sole chromosomal anomaly in hematologic neoplasms. Cancer Genet Cytogenet. 1994;74(1):62-65.

21. Dastugue N, Lafage-Pochitaloff M, Pages MP, et al. Cytogenetic profile of childhood and adult megakaryoblastic leukemia (M7): a study of the Groupe Francais de Cytogenetique Hematologique (GFCH). Blood. 2002;100(2):618-626.

22. Dang J, Nance S, Ma J, et al. AMKL chimeric transcription factors are potent inducers of leukemia. Leukemia. 2017;31(10):2228-2234.

23. Lebert-Ghali CE, Neault M, Fournier M, et al. Generation of a novel mouse model recapitulating features of human acute megakaryoblastic leukemia. Exp Hematol. 2018;64:S79.

24. Wildenhain S, Ingenhag D, Ruckert C, et al. Homeobox protein HB9 binds to the prostaglandin E receptor 2 promoter and inhibits intracellular cAMP mobilization in leukemic cells. J Biol Chem. 2012;287 (48):40703-40712.

\title{
New insights into the causes of thrombotic events in patients with myeloproliferative neoplasms raise the possibility of novel therapeutic approaches
}

\author{
Michal Bar-Natan and Ronald Hoffman \\ Tisch Cancer Institute, Icahn School of Medicine at Mount Sinai, New York, NY, USA
}

E-mail: ronald.hoffman@mssm.edu

doi:10.3324/haematol.2018.205989

T The Philadelphia chromosome-negative myeloproliferative neoplasms (MPN) include polycythemia vera (PV), essential thrombocythemia (ET) and primary myelofibrosis (MF). This group of clonal hematological malignancies is associated with a protracted clinical course frequently punctuated by thrombotic events. Such thrombotic events have been previously attributed to excessive numbers of functionally abnormal red cells, platelets and leukocytes. MPN patients are not only at a high risk of developing arterial and venous thromboses, but also throm- 